\title{
Germinação e crescimento inicial de mudas de espécies não convencionais de fisális em diferentes substratos e ambientes
}

\author{
Germination and initial growth of non-conventional species of physalis on different substrates and \\ environments
}

\author{
Rafael Machado de Cecco ${ }^{1}$, Elcio Silvério Klosowski ${ }^{1}$, Daniel Fernandes da Silva ${ }^{2 \star} \&$ Fabíola Villa ${ }^{1}$ \\ ${ }^{1}$ Universidade Estadual do Oeste do Paraná, Marechal Cândido Rondon, PR, Brasil. *Autor para correspondência: \\ daniel_eafi@yahoo.com.br. \\ ${ }^{2}$ Universidade Federal de Lavras, Lavras, MG, Brasil.
}

Submissão: 14/02/2017 | Aceite: 10/12/2017

\begin{abstract}
RESUMO
A utilização de substratos e ambientes é importante fator a ser analisado na determinação da qualidade de germinação e crescimento de mudas de espécies de fisális. Diante do exposto, objetivou-se com o presente trabalho, avaliar a germinação e o crescimento inicial de duas espécies não convencionais de fisális, submetidas a diferentes ambientes e substratos. Conduziu-se dois experimentos simultâneos no período de março a maio/2014, na Unioeste, PR. O delineamento experimental utilizado no primeiro experimento foi blocos casualizados, fatorial 2x3 (2 espécies de fisális: Physalis minima, Physalis ixocarpa x 3 ambientes: ambiente protegido com cobertura plástica de $150 \mu \mathrm{m}$; telado com $50 \%$ de sombreamento e pleno sol), contendo 3 repetições e 50 sementes por parcela experimental. O delineamento do segundo experimento foi blocos casualizados, fatorial 2x4 (2 espécies de fisális: Physalis minima, Physalis ixocarpa x 4 substratos: vermiculita expandida de textura média + Latossolo; Húmus Fértil ${ }^{\circledR}+$ Latossolo; areia lavada de textura fina + Latossolo e Húmus Fértil ${ }^{\circledR}$ + vermiculita expandida de textura média, na proporção $1: 1 \mathrm{v}: \mathrm{v})$, contendo 3 repetições e 32 sementes por parcela experimental. Monitorou-se os experimentos por 22 dias, para obtenção do índice de velocidade de emergência. Aos 60 dias após a semeadura avaliou-se a altura das plântulas, diâmetro do caule, número de folhas, biomassa fresca e seca das plântulas. Estufa agrícola e telado propiciam índice de velocidade de emergência de sementes de Physalis ixocarpa e Physalis mínima, sem distinção entre esses ambientes. Mudas de $P$. ixocarpa devem ser produzidas em estufa, utilizando-se como mistura de substrato, Húmus Fértil ${ }^{\circledR}+$ vermiculita expandida de textura média, ou Latossolo + vermiculita. Mudas de $P$. minima devem ser produzidas em mistura de Húmus Fértil ${ }^{\circledR}$ + vermiculita, sob estufa ou telado.
\end{abstract}

PALAVRAS-CHAVE: Physalis minima L., Physalis ixocarpa Brot., pequenas frutas, reprodução sexuada.

\begin{abstract}
The use of substrates and environments is an important factor to be considered in determining the quality of germination and seedling growing of species of physalis. Given the above, the objective with this study was to evaluate the germination and early crowing of two non-conventional species of physalis, subjected to different environments and substrates. Were conducted two simultaneous experiments in the period from March to May / 2014 in Unioeste, PR. The experimental design used in the first experiment was a randomized, $2 \times 3$ factorial (2 physalis species: Physalis minima, Physalis ixocarpa x 3 environments: protect environment with plastic cover of $150 \mu \mathrm{m}$, greenhouse with $50 \%$ of shading and full sun) blocks containing 3 replicates and 50 seeds per experimental plot. The design of the second experiment was randomized, 2x4 factorial (2 physalis species: Physalis minima, Physalis ixocarpa $\times 4$ substrates: expanded vermiculite of medium texture + latossol, Húmus Fértil ${ }^{\circledR}+$ latossol, washed sand of fine texture + latossol and Humus Fértil ${ }^{\circledR}+$ expanded vermiculite of medium texture, in proportion 1:1 v:v) blocks containing three replicates of 32 seeds per experimental plot. Was monitored by 22 days the experiments to obtain the emergence speed index. 60 days after the sowing was evaluated seedling height, stem diameter, number of leaves, fresh and dry biomass of seedlings. We conclude that, agricultural greenhouse and greenhouse of shade net providing emergence speed index of Physalis minima and Physalis Ixocarpa without distinction between these environments. Seedlings of $P$. ixocarpa should be produced in agricultural greenhouse, using as substrate mix of Humus fértil ${ }^{\circledR}$ commercial substrate + expanded vermiculite or latosol + vermiculite. Seedlings of $P$. minima must be
\end{abstract}


produced in a mixture of Humus Fértil ${ }^{\circledR}+$ vermiculite under agricultural greenhouse with or under shade net.

KEYWORDS: Physalis minima L., Physalis ixocarpa Brot., small fruits, sexual reproduction.

\section{INTRODUÇÃO}

A produção de pequenos frutos, de modo geral, caracteriza-se pelo baixo custo de implantação, custo de produção acessível aos pequenos produtores, bom retorno econômico, boa adaptação às condições socioeconômicas e do ambiente local, grande exigência de mão de obra, possibilidade de cultivo em sistema orgânico e demanda maior do que oferta (RODRIGUES et al. 2009).

Dentre os pequenos frutos, a fisális é uma das plantas que ocupa espaço considerável de produção no sul e sudeste do país (LIMA et al. 2010, SILVA et al. 2013). Além do fruto como produto principal, o alto valor agregado se deve a utilização das raízes e folhas no mercado farmacológico, e do cálice, em decorações (MUNIZ et al. 2014). Sua propagação a nível comercial se dá via sexuada, por meio do uso de sementes retiradas de frutos maduros (85-90\%) (SBRUSSI et al. 2014) e a formação de novas mudas da espécie tem demanda contínua e crescente em função do caráter anual no cultivo da espécie (SILVA et al. 2017).

Independentemente do método de propagação adotado, a primeira etapa de produção é o desenvolvimento de mudas. Para que esse processo inicial seja otimizado, muitas vezes se faz necessário o uso de materiais alternativos ao solo, seja por exigências de ordem física ou fisiológica das plantas, sendo nestes casos, utilizados os substratos (FERNANDES et al. 2006).

A escolha do(s) material(is) para compor um substrato deve levar em consideração as características físico-químicas, tais como, densidade, capacidade de economia hídrica, aeração, permeabilidade, poder de tamponamento, capacidade de retenção de nutrientes, espécie a ser cultivada, além dos aspectos econômicos, como baixo custo e disponibilidade (KRATZ et al. 2013).

Diversos materiais orgânicos e inorgânicos têm sido utilizados no preparo de substratos, na produção de mudas frutíferas. Muitas vezes, para atender a todos os aspectos citados anteriormente, se faz necessário a utilização de mais de um material para compor um substrato de boa qualidade (FERRAZ et al. 2005).

No Brasil, há certa escassez de trabalhos utilizando ambientes na produção de mudas de qualidade de pequenos frutos, existindo poucas fontes científicas argumentando sobre qual a melhor estrutura a ser empregada. Em relação ao fisális, a falta de informações sobre sistemas de produção de mudas, tem levado os fruticultores a adotar o mesmo sistema usado no cultivo do tomateiro (LIMA et al. 2010), devido à proximidade taxonômica das espécies, onde ambas pertencem à mesma família e têm hábito de crescimento similar.

Sabendo-se que em cada região do país ocorrem características de intensidade luminosa, temperatura, e umidade relativa do ar distinta, objetivou-se com o presente estudo avaliar o crescimento inicial de mudas de duas espécies não convencionais de fisális, empregando diversos substratos e ambientes favoráveis que propiciem o crescimento adequado destas.

\section{MATERIAL E MÉTODOS}

Conduziu-se dois experimentos no período de março a maio/2014, na Estação de Horticultura e Controle Biológico "Professor Mário César Lopes", que se encontra na longitude $54^{\circ} 22^{\prime} \mathrm{W}$, latitude $24^{\circ} 46^{\prime} \mathrm{S}$ e altitude de $420 \mathrm{~m}$. A Estação pertence ao Núcleo de Estações Experimentais (NEE) da Universidade Estadual do Oeste do Paraná (Unioeste), Campus Marechal Cândido Rondon, PR.

O clima da região, segundo a classificação de Köppen, é tipo Cfa, subtropical, com média anual de precipitação entre 1600-1800 mm e umidade relativa entre $70-75 \%$. A média anual de temperatura do ar encontra-se na faixa de $22-23^{\circ} \mathrm{C}$ (CAVIGLIONE et al. 2000).

O material vegetal utilizado na implantação dos experimentos foram sementes de duas espécies de fisális (Physalis minima e Physalis ixocarpa), obtidas de cultivo na safra 2013/2014, em pomar experimental da Universidade Federal de Lavras (UFLA), MG. As sementes foram coletadas de frutos completamente maduros, extraídas com o auxílio de liquidificador e, posteriormente, secas à sombra.

Conduziu-se dois experimentos simultaneamente. O primeiro, com delineamento experimental em blocos ao acaso, esquema fatorial 2×3 (duas espécies de fisális, Physalis minima e Physalis ixocarpa) $\times 3$ ambientes, sendo o ambiente protegido com cobertura plástica de $150 \mu \mathrm{m}$; telado com $50 \%$ de absorção de radiação solar e a pleno sol, contendo 3 repetições e 50 sementes por parcela experimental. $O$ substrato 
em que as sementes foram acondicionadas foi Latossolo + vermiculita expandida de textura média + Húmus Fértil ${ }^{\circledR}$, na proporção 1:1:1, à base de volume.

O segundo experimento foi conduzido com delineamento experimental em blocos ao acaso, esquema fatorial $2 \times 4$ (duas espécies de fisális, sendo $P$. minima e $P$. ixocarpa) $\times(4$ substratos, sendo vermiculita expandida de textura média + Latossolo; Húmus Fértil ${ }^{\circledR}$ + Latossolo; areia lavada de textura fina + Latossolo e Húmus Fértil ${ }^{\circledR}$ + vermiculita expandida de textura média, todos na proporção 1:1, à base de volume), contendo 4 repetições e 32 sementes por parcela experimental.

Realizou-se a semeadura para ambos os experimentos em bandejas de poliestireno expandido (200 células para o primeiro experimento e 128 células para o segundo experimento). A irrigação foi realizada diariamente com auxílio de mangueira com ponteira de microaspersão e regador.

Durante os primeiros 22 dias, contou-se diariamente o número de sementes germinadas para avaliação do Índice de Velocidade de Emergência (IVE), levando em consideração a estabilização da emergência em ambas as espécies. O Índice de Velocidade de Emergência (IVE) foi calculado pela equação proposta por MAGUIRE (1962):

$\operatorname{IVE}=\mathrm{N}_{1} / \mathrm{D}_{1}+\mathrm{N}_{2} / \mathrm{D}_{2}+\mathrm{Nn} / \mathrm{Dn}$

em que: $N_{1}, N_{2}, N n$ = número de plântulas emergidas na primeira, segunda, e sucessivamente até a última contagem, $\mathrm{D}_{1}, \mathrm{D}_{2}, \mathrm{Dn}=$ número de dias da semeadura na primeira, segunda, até a última contagem.

Aos 40 dias após o plantio, realizou-se adubação em cobertura com ureia, visando favorecer o crescimento das plantas e a cor verde-escura das mesmas, promover o desenvolvimento do sistema radicular, melhorando a absorção de nutrientes presentes na solução do solo.

A segunda avaliação foi realizada após 60 dias da implantação dos experimentos, onde avaliou-se a altura de plantas $(\mathrm{cm})$, diâmetro do caule $(\mathrm{mm})$, número de folhas, biomassa fresca $(\mathrm{g})$ e seca $(\mathrm{g})$ das plantas. A altura foi determinada com régua graduada, e a medida do diâmetro do caule padronizada para as plantas a $2 \mathrm{~cm}$ do solo, foi obtida com auxílio de paquímetro digital. $O$ número de folhas foi contado manualmente e as mensurações de biomassa fresca e seca foram realizadas em laboratório. As raízes foram lavadas em água corrente para a retirada de todas as partículas de substrato, e pesadas em balança analítica, para obtenção da biomassa fresca em gramas $(\mathrm{g})$. Imediatamente após a pesagem, as plantas foram acondicionadas em sacos de papel $\mathrm{Kraft}^{\circledR}$, secas em estufa, com circulação de ar a $65^{\circ} \mathrm{C}$, durante 72 horas. Posteriormente, mensurou-se a biomassa seca das mudas.

Os dados obtidos foram submetidos à análise da variância, sendo as médias comparadas pelo teste de Scott-Knott, a 5\% de probabilidade de erro, com o auxílio do programa estatístico Sisvar (FERREIRA 2011).

\section{RESULTADOS E DISCUSSÃO}

Nas Figuras 1 e 2 observa-se as temperaturas médias, máximas e mínimas semanais $\left({ }^{\circ} \mathrm{C}\right)$ e umidade relativa do ar durante o período de realização dos experimentos. Os dados foram obtidos com o auxílio de equipamentos meteorológicos instalados na Estação Experimental Automática de Marechal Cândido Rondon (convênio INMET/Unioeste), auxiliando no esclarecimento dos resultados do estudo.

A partir dos dados observados nas Figuras 1 e 2, verifica-se oscilações entre temperatura e umidade relativa acentuadas ao longo do período de realização dos experimentos, explicando assim, a germinação inferior, bem como o reduzido desenvolvimento de mudas cultivadas a pleno sol, em relação aos demais ambientes que, devido à cobertura plástica e sombrite, têm estas oscilações reduzidas.

Alguns fatores são, de certa forma, controlados em ambiente protegido (VIDA et al. 2004). Desta maneira, possibilita-se a proteção das plantas contra condições adversas de clima e solo, como ventos, evapotranspiração, umidade relativa do ar e solo, baixas temperaturas, radiação solar, efeito direto das chuvas, lixiviação de nutrientes, aeração do solo, chuvas de granizo, dentre outros fatores (ANDRIOLO 2011).

As sementes são capazes de germinar sob uma amplitude de temperatura definida para cada espécie (GENDREAU \& CORBINEAU 2009). Os mesmos autores afirmam que existe uma temperatura ótima, mínima e máxima para a germinação, sendo ótima aquela em que a mais alta percentagem de germinação é obtida no menor espaço de tempo. A temperatura ótima para a maioria das espécies encontra-se entre 15-30 ํㅡ e a máxima entre 35-40 ํㅡ (MARCOS FILHO 2005). 


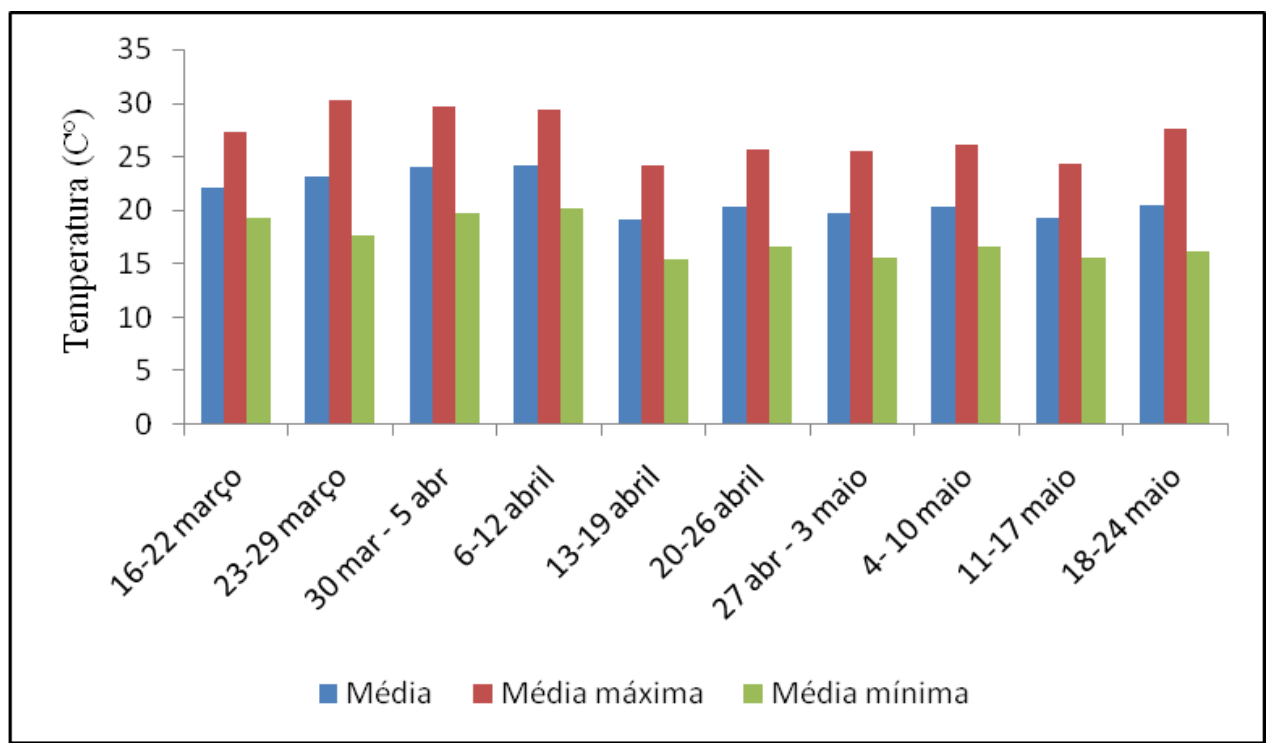

Figura 1. Temperaturas médias, médias máximas e médias mínimas semanais da temperatura do ar $\left({ }^{\circ} \mathrm{C}\right)$, durante o período de realização dos experimentos. Unioeste, Campus Marechal Cândido Rondon, PR. 2016.

Figure 1. Average temperatures, average maximum and average minimum weekly air temperature $\left({ }^{\circ} \mathrm{C}\right)$ during the period of the experiments. Unioeste, Campus Marechal Cândido Rondon, PR. 2016.

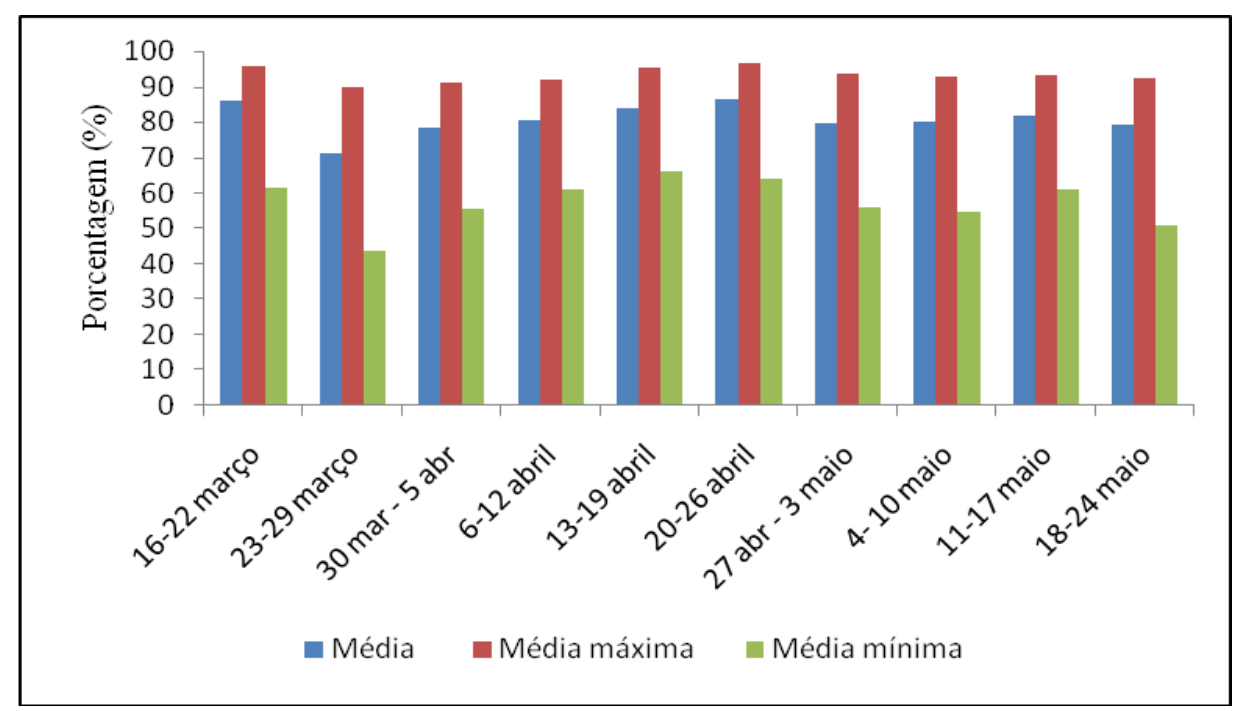

Figura 2. Umidades relativas do ar (máximas, médias e mínimas) medidas semanalmente durante o período de realização do experimento. Unioeste, Campus Marechal Cândido Rondon, PR. 2016.

Figure 2. Relative humidity of the air (maximum, medium and minimum) measured weekly during the experiment period of realization. Unioeste, Campus Marechal Cândido Rondon, PR. 2016.

\section{Experimento 1}

Na Tabela 1 observa-se que houve significância apenas para ambiente em relação a porcentagem de emergência e IVE, sendo estes fatores, afetados pelos ambientes utilizados. Para estes parâmetros, o cultivo em ambiente protegido sob cobertura plástica e telado foi significativamente superior quando comparado ao cultivo a pleno sol.

Sabe-se que, enquanto as sementes estão abrigadas no substrato, oscilações de temperatura e umidade relativa do ar não são tão prejudiciais para a germinação das mesmas, pois o substrato serve de proteção às sementes contra os efeitos adversos provocados pelo ambiente térmico. Pode-se afirmar então, que a pleno sol, a germinação das duas espécies de fisális estudadas, apesar da significância estatística ser menor quando comparada aos outros ambientes, não foi limitante na germinação e emergência. 
Contudo, à medida que as plantas começam a emergir, analisando variáveis observadas neste trabalho, pode-se afirmar que o ambiente climático interferiu negativamente no crescimento das mesmas.

Tabela 1. Percentagem de emergência de sementes de Physalis ixocarpa e Physalis minima, cultivadas em três ambientes. Unioeste, Campus Marechal Cândido Rondon, PR. 2016.

Table 1. Percentage emergency of Physalis ixocarpa and Physalis minima seeds, grown in three environments. Unioeste, Campus Marechal Cândido Rondon, PR. 2016.

\begin{tabular}{lcc}
\hline Ambientes de cultivo & $\begin{array}{c}\text { Percentagem } \\
\text { de emergência }\end{array}$ & IVE \\
\hline Ambiente protegido & $87,0 \mathrm{a}^{*}$ & $50,61 \mathrm{a}$ \\
Telado & $89,0 \mathrm{a}$ & $47,36 \mathrm{a}$ \\
Pleno sol & $73,3 \mathrm{~b}$ & $29,41 \mathrm{~b}$ \\
\hline $\mathrm{CV}(\%)$ & 11,59 & 10,10 \\
\hline
\end{tabular}

${ }^{*}$ Médias seguidas pela mesma letra minúscula na coluna não diferem entre si pelo teste de Skott-Knott, a $5 \%$ de probabilidade de erro.

Contrariamente, SILVA et al. (2016) não observaram variação na porcentagem de emergência em diferentes ambientes de germinação na cidade de Lavras, Minas Gerais, sendo a porcentagem de emergência significativamente diferente somente entre espécies de fisális estudadas.

O IVE é um índice calculado a partir dos dados de contagem de plantas emergidas e tem como objetivo estabelecer diferenças na velocidade de emergência de sementes cultivadas. Para a estufa com cobertura plástica e telado, o IVE calculado não mostrou diferença estatística, sendo este considerado ideal quanto à velocidade de emergência, por ocorrer em curto espaço de tempo, demonstrando condições suficientemente propícias à expressão do vigor das sementes. O ambiente a pleno sol mostrou-se ineficiente na emergência de sementes de fisális (Tabela 1).

A temperatura em que ocorre a germinação é um fator muito importante, que exerce grande influência no processo, tanto na germinação total, quanto na velocidade de germinação (CARVALHO \& NAKAGAWA 2000). Resultados encontrados no presente estudo corroboram com REIS et al. (2010), que estudaram os mesmos ambientes para emergência de amêndoas de pessegueiro cultivar Okinawa, e para os quais obtiveram resultados significativamente superiores para emergência e velocidade de emergência em casa-de-vegetação, seguido de telado e céu aberto.

Os resultados encontrados para o IVE também estão de acordo com SILVA et al. (2016) e OLIVEIRA et al. (2009) que encontraram menor IVE de sementes de Copernica hospita Martius e Physalis peruviana L. em ambiente a pleno sol, quando comparado a ambientes protegidos. Estes autores explicam que o IVE a pleno sol, é reduzido devido a efeitos maléficos de altas temperaturas, principalmente a desidratação excessiva do substrato, que desta forma, reduz a velocidade do processo de embebição da semente e, consequentemente, a germinação.

Na Tabela 2 pode-se observar interação significativa para altura e diâmetro médio de colo entre espécies e ambiente de cultivo. Valores superiores foram verificados na altura média das mudas de Physalis ixocarpa em ambiente protegido com cobertura plástica, ou seja, mudas maiores e mais vigorosas foram obtidas neste local. Este resultado pode ser explicado pela baixa oscilação de temperatura e umidade relativa comum neste tipo de ambiente.

Para $P$. ixocarpa, maior diâmetro de colo foi verificado em ambiente protegido. O cultivo a pleno sol para esta espécie mostrou-se inadequado para a produção de mudas. Isso se deve a grande amplitude de temperatura e umidade que ocorrem durante o dia, causando estresse e promovendo menor desenvolvimento das mudas de fisális.

O aumento do diâmetro do colo em $P$. ixocarpa seguiu os mesmos padrões observados para 0 aumento do comprimento das mudas, sendo superior em plantas cultivadas em ambiente protegido. Para $P$. minima, o diâmetro do colo distinguiu-se dos demais nos tratamentos a pleno sol e sob condições de telado, sendo os dois ambientes considerados propícios para o desenvolvimento desta espécie.

O aumento do diâmetro do caule acompanhando um maior crescimento da muda, como observado em $P$. ixocarpa sob cobertura plástica, é uma característica desejável, pois garante maior sustentação à parte aérea da nova planta em formação. Este aumento do diâmetro do caule em diferentes ambientes de cultivo, acompanhando um incremento na altura da muda, pode ser observado, por exemplo, em mudas de alecrim e pau ferro, em diferentes ambientes de cultivo (LIMA et al. 2008, SOUZA et al. 2014). Todavia, 
existem espécies que não modificam seu diâmetro de caule independente da mudança no ambiente de cultivo, como a melissa e o tapeinóquilo (MEIRA et al. 2012).

Tabela 2. Altura média de mudas (AMP), diâmetro médio de caule (DMC), número médio de folhas (NMF) de Physalis ixocarpa (PI) e Physalis minima (PM), cultivadas em ambiente protegido com cobertura plástica (APCP), telado (T) e a pleno sol (PS). Unioeste, Campus Marechal Cândido Rondon, PR. 2016.

Table 2. Average height of seedlings (AMP), average stem diameter (DMC), average number of leaves (NMF) of Physalis ixocarpa (PI) and Physalis minima (PM), grown in protected environment with plastic cover (APCP), greenhouse with shade net (T) and full sun (PS). Unioeste, Campus Marechal Cândido Rondon, PR. 2016.

\begin{tabular}{lcccccc}
\hline $\begin{array}{l}\text { Ambientes de } \\
\text { cultivo }\end{array}$ & \multicolumn{2}{c}{ AMP $(\mathrm{cm})$} & \multicolumn{2}{c}{ DMC $(\mathrm{mm})$} & \multicolumn{3}{c}{ NMF } \\
\hline & $\mathrm{PI}$ & $\mathrm{PM}$ & $\mathrm{PI}$ & $\mathrm{PM}$ & $\mathrm{PI}$ & $\mathrm{PM}$ \\
\hline APCP & $15,96 \mathrm{aA} A^{*}$ & $6,45 \mathrm{bA}$ & $2,03 \mathrm{aA}$ & $1,74 \mathrm{bA}$ & $9,73 \mathrm{aA}$ & $8,13 \mathrm{bA}$ \\
$\mathrm{T}$ & $11,73 \mathrm{aB}$ & $6,67 \mathrm{bA}$ & $1,64 \mathrm{aB}$ & $1,70 \mathrm{aA}$ & $7,00 \mathrm{aB}$ & $7,13 \mathrm{aA}$ \\
PS & $4,53 \mathrm{aC}$ & $3,77 \mathrm{aB}$ & $1,48 \mathrm{bB}$ & $1,85 \mathrm{aA}$ & $4,17 \mathrm{bC}$ & $7,20 \mathrm{aA}$ \\
\hline
\end{tabular}

CV (\%) $\quad 16,93 \quad 5,66 \quad 9,97$

${ }^{*}$ Médias seguidas pela mesma letra minúscula na linha, comparando as espécies de fisális para cada ambiente de cultivo, e maiúscula na coluna, comparando os ambientes de cultivo dentro de cada espécie de fisális não diferem entre si pelo teste de Skott-Knott, a 5\% de probabilidade de erro.

Conforme Tabela 2, observou-se interação significativa para número de folhas em relação às espécies de fisális e ambiente de cultivo. Os diversos ambientes para Physalis ixocarpa diferiram estatisticamente entre si, ao passo que, para $P$. mínima, não houve diferença significativa entre os mesmos. Tal condição evidencia uma possível condição de rusticidade mais acentuada em Physalis minima em relação a $P$. ixocarpa.

Maior número de folhas foi verificado em ambiente protegido para $P$. ixocarpa e telado e pleno sol para $P$. minima. Além da diferença entre genótipos, esta resposta das espécies pode estar relacionada a uma adaptação ecológica à condição de baixa luminosidade, para que a planta obtenha luz suficiente para desempenho de seus processos vitais (LIMA JUNIOR et al. 2005).

Conforme Tabela 3, verifica-se interação significativa para biomassa fresca e seca das mudas das espécies de fisális.

Tabela 3. Biomassa fresca (BMF) e biomassa seca (BMS) de mudas de Physalis ixocarpa e Physalis minima, cultivadas em ambiente protegido com cobertura plástica (APCP), telado (T) e pleno sol (PS). Unioeste, Campus Marechal Cândido Rondon, PR. 2016.

Table 3. Fresh biomass (BMF) and dry biomass (BMS) seedlings of Physalis ixocarpa and Physalis minima, grown in protected environment with plastic cover (APCP), greenhouse with shade net $(T)$ and full sun (PS). Unioeste, Campus Marechal Cândido Rondon, PR. 2016.

\begin{tabular}{lcccc}
\hline \multirow{2}{*}{ Ambientes de cultivo } & \multicolumn{2}{c}{ Physalis ixocarpa } & \multicolumn{2}{c}{ Physalis minima } \\
\cline { 2 - 5 } & $\mathrm{BMF}(\mathrm{g})$ & $\mathrm{BMS}(\mathrm{g})$ & $\mathrm{BMF}(\mathrm{g})$ & $\mathrm{BMS}(\mathrm{g})$ \\
\hline APCP & $0,857 \mathrm{aA} \mathrm{A}^{*}$ & $0,100 \mathrm{aA}$ & $0,336 \mathrm{bA}$ & $0,053 \mathrm{bA}$ \\
T & $0,588 \mathrm{aB}$ & $0,062 \mathrm{aB}$ & $0,416 \mathrm{bA}$ & $0,043 \mathrm{bA}$ \\
PS & $0,187 \mathrm{bC}$ & $0,023 \mathrm{bC}$ & $0,361 \mathrm{aA}$ & $0,059 \mathrm{aA}$ \\
\hline CV $(\%)$ & 16,00 & 13,83 & 16,00 & 13,83
\end{tabular}

*Médias seguidas pela mesma letra minúscula na linha, comparando as biomassas fresca e seca das espécies de fisális dentro de cada ambiente de cultivo, e maiúscula na coluna comparando os ambientes de cultivo e biomassas fresca e seca para cada espécie de fisális, não diferem entre si pelo teste de Skott-Knott, a 5\% de probabilidade de erro.

A biomassa fresca (BMF) e biomassa seca (BMS) são consequências diretas do crescimento vegetal, desta forma, plantas que apresentam crescimento acentuado em altura e número de folhas principalmente, tendem a apresentar maior biomassa fresca e seca. Tal resultado é confirmado na Tabela 3 , onde se verifica valores de $0,857 \mathrm{~g}$ de BMF e $0,100 \mathrm{~g}$ de BMS em mudas de $P$. ixocarpa cultivadas em ambiente 
protegido. Neste ambiente foram obtidas mudas de fisális com maior comprimento, diâmetro de caule e número de folhas.

Maior teor de BMF e BMS pode ser explicado pelo fato do ambiente protegido ser difusor de luz, promovendo maior crescimento das mudas. Neste ambiente, devido ao sombreamento, observa-se menor amplitude de temperatura e umidade relativa do ar, permitindo assim, que as mudas realizassem fotossíntese com maior eficiência, gerando maior acúmulo de biomassa seca nas folhas.

\section{Experimento 2}

Os resultados obtidos para percentagem de emergência de fisális nos substratos demonstrou significância apenas para as espécies (Tabela 4).

Tabela 4. Percentagem de emergência de sementes de Physalis ixocarpa e Physalis minima. Unioeste, Campus Marechal Cândido Rondon, PR. 2016.

Table 4. Percentage of emergency of Physalis ixocarpa and Physalis minima seeds. Unioeste, Campus Marechal Cândido Rondon, PR. 2016.

\begin{tabular}{lc}
\hline Ambientes de cultivo & Percentagem de emergência \\
\hline Physalis minima & $80,21 \mathrm{a}^{*}$ \\
Physalis ixocarpa & $65,11 \mathrm{~b}$ \\
\hline CV (\%) & 15,77 \\
\hline
\end{tabular}

${ }^{\star}$ Médias seguidas pela mesma letra minúscula na coluna não diferem entre si pelo teste de Skott-Knott, a $5 \%$ de probabilidade de erro.

Tal diferença entre as espécies, provavelmente está ligada às condições genéticas de cada uma, sendo que a emergência de sementes é uma característica relacionada à capacidade de colonização de um ambiente, por uma determinada espécie, variando amplamente entre espécies e gêneros (RUFATO et al. 2008).

Na Tabela 5 verifica-se interação significativa para altura das mudas, diâmetro médio de caule e número médio de folhas das espécies de fisális nos substratos.

Tabela 5. Altura média de mudas (AMP), diâmetro médio de caule (DMC), número médio de folhas (NMF) de Physalis ixocarpa (PI) e Physalis minima (PM), cultivadas em substratos Latossolo + vermiculita $(L+V)$, Húmus Férti ${ }^{\circledR}+$ Latossolo $(H F+L)$, Latossolo + areia $(L+A)$, Húmus Fértil ${ }^{\circledR}+$ vermiculita $^{2}$ $(\mathrm{HF}+\mathrm{V})$. Unioeste, Campus Marechal Cândido Rondon, PR. 2016.

Table 5. Average height of seedlings (AMP), average stem diameter (DMC), average number of leaves (NMF) of Physalis ixocarpa (PI) and Physalis minima (PM), grown in substrates latosol + ermiculite $(L+V)$, Humus Férti ${ }^{\oplus}+$ latosol $(H F+L)$, latosol + sand $(L+A)$, Humus Fértil ${ }^{\circledR}+$ vermiculite $(H F+$ V). Unioeste, Campus Marechal Cândido Rondon, PR. 2016.

\begin{tabular}{lcccccc}
\hline Substratos & \multicolumn{2}{c}{ AMP $(\mathrm{cm})$} & \multicolumn{2}{c}{ DMC $(\mathrm{mm})$} & \multicolumn{2}{c}{ NMF } \\
\hline & $\mathrm{PI}$ & $\mathrm{PM}$ & $\mathrm{PI}$ & $\mathrm{PM}$ & $\mathrm{PI}$ & $\mathrm{PM}$ \\
\hline $\mathrm{L}+\mathrm{V}$ & $27,03 \mathrm{aA} A^{*}$ & $6,79 \mathrm{bC}$ & $2,76 \mathrm{aA}$ & $1,74 \mathrm{bB}$ & $9,13 \mathrm{aA}$ & $7,47 \mathrm{aB}$ \\
$\mathrm{HF}+\mathrm{L}$ & $17,31 \mathrm{aB}$ & $18,62 \mathrm{aB}$ & $1,91 \mathrm{aB}$ & $3,03 \mathrm{bA}$ & $7,40 \mathrm{bB}$ & $10,53 \mathrm{aA}$ \\
$\mathrm{L}+\mathrm{A}$ & $12,35 \mathrm{aC}$ & $7,03 \mathrm{bC}$ & $1,64 \mathrm{aB}$ & $1,55 \mathrm{aB}$ & $6,40 \mathrm{aB}$ & $7,73 \mathrm{aB}$ \\
$\mathrm{HF}+\mathrm{V}$ & $26,93 \mathrm{aA}$ & $22,99 \mathrm{bA}$ & $2,90 \mathrm{aA}$ & $2,98 \mathrm{aA}$ & $9,07 \mathrm{aA}$ & $10,47 \mathrm{aA}$ \\
\hline $\mathrm{CV}(\%)$ & \multicolumn{3}{c}{12,15} & \multicolumn{2}{c}{7,79} & \multicolumn{2}{c}{11,51} \\
\hline
\end{tabular}

*Médias seguidas pela mesma letra minúscula na linha, comparando as espécies de fisális para cada substrato, e maiúscula na coluna, comparando os substratos dentro de cada espécie de fisális, não diferem entre si pelo teste de Skott-Knott, a $5 \%$ de probabilidade de erro.

Em relação à altura das mudas, para a espécie $P$. ixocarpa, os substratos que continham vermiculita apresentaram maior altura de mudas, corroborando trabalhos com Physalis peruviana realizado por PIVA et al. (2013). Para $P$. minina resultados superiores foram verificados na mistura de Húmus Fértil ${ }^{\circledR}+$ vermiculita. $^{2}$ A vermiculita tem como características principais, a baixa densidade e elevada porosidade (FACHINELLO et al. 2005). De acordo com HARTMANN et al. (2011), o sistema radicular só se desenvolve 
satisfatoriamente quando o substrato combina boa aeração com alta capacidade de retenção de água, boa drenagem e ausência de contaminantes, o que, em consequência, gera um bom desenvolvimento da parte aérea das mudas.

O desempenho inadequado do substrato Latossolo + areia para ambas as espécies é explicado por BRAUN et al. (2010), que afirmam que, tanto o solo como a areia, possuem alta densidade, com partículas compactas e baixa porosidade. Os substratos que possuem comportamento singular, quando condicionados a um espaço limitado (no caso de vasos, tubetes e sacos de polietileno), assim como o solo, também influenciam no desenvolvimento das mudas, na arquitetura do sistema radicular e nas associações biológicas das plântulas com o meio, estando prioritariamente relacionados à nutrição e translocação de água no sistema planta-atmosfera.

Em relação ao diâmetro do colo e número de folhas (Tabela 5) das mudas de $P$. ixocarpa e P. minima cultivadas nos substratos, a espécie $P$. ixocarpa apresentou resultados superiores em substrato que continha vermiculita. Para $P$. minima, melhores resultados foram verificados em substrato a base de Húmus Fértil ${ }^{\circledR}$. A formulação do substrato com combinações de vários materiais permite a obtenção de um produto final com características químicas e físicas mais favoráveis ao desenvolvimento de plantas, o que possibilita a obtenção de mudas com qualidade morfofisiológica superior (PELIZZA et al. 2013).

Os componentes de um substrato devem apresentar características favoráveis ao desenvolvimento de plântulas. Tais características podem variar de acordo com a espécie. Desta forma, por ser a vermiculita uma fonte inerte combinada ao Latossolo ou substrato comercial, pode ter contribuído para a formação de um substrato mais aerado com capacidade de retenção de água mais apropriada para a formação de mudas de $P$. ixocarpa. Por outro lado, $P$. minima pode ter exigências nutricionais mais elevadas, exigindo substratos mais ricos em nutrientes, não bastando somente os componentes do solo para seu desenvolvimento, sendo favorecido pela utilização de substratos comerciais, como o Húmus Fértil ${ }^{\circledR}$, caracterizado por apresentar maior uniformidade física e química (FACHINELLO et al. 2005).

A biomassa fresca e seca das mudas reflete diretamente nos resultados obtidos nas variáveis anteriormente analisadas (Tabela 6). Mudas com maior massa para $P$. ixocarpa e $P$. mínima foram observados em substrato contendo vermiculita.

Tabela 6. Biomassa fresca (BMF) e biomassa seca (BMS) de mudas de Physalis ixocarpa e Physalis minima, cultivadas em diferentes substratos. Unioeste, Campus Marechal Cândido Rondon, PR. 2016.

Table 6. Fresh biomass (BMF) and dry biomass (BMS) seedlings of Physalis ixocarpa and Physalis minima, grown on different substrates. Unioeste, Campus Marechal Cândido Rondon, PR. 2016.

\begin{tabular}{lccrc}
\hline \multirow{2}{*}{ Substratos } & \multicolumn{2}{c}{$P$. ixocarpa } & \multicolumn{2}{c}{ P. minima } \\
\cline { 2 - 5 } & $\mathrm{BMF}(\mathrm{g})$ & $\mathrm{BMS}(\mathrm{g})$ & $\mathrm{BMF}(\mathrm{g})$ & $\mathrm{BMS}(\mathrm{g})$ \\
\hline $\mathrm{L}+\mathrm{V}$ & $11,41 \mathrm{aA} A^{*}$ & $1,40 \mathrm{aA}$ & $3,46 \mathrm{bC}$ & $0,38 \mathrm{bC}$ \\
$\mathrm{HF}+\mathrm{L}$ & $4,20 \mathrm{bB}$ & $0,42 \mathrm{bB}$ & $10,74 \mathrm{aB}$ & $1,28 \mathrm{aB}$ \\
$\mathrm{A}+\mathrm{L}$ & $2,38 \mathrm{aB}$ & $0,32 \mathrm{aB}$ & $3,08 \mathrm{aC}$ & $0,37 \mathrm{aC}$ \\
$\mathrm{HF}+\mathrm{V}$ & $12,28 \mathrm{aA}$ & $1,48 \mathrm{bA}$ & $15,80 \mathrm{aA}$ & $1,81 \mathrm{aA}$ \\
\hline $\mathrm{CV}(\%)$ & 17,59 & 16,12 & 17,59 & 16,12 \\
\hline
\end{tabular}

*Médias seguidas pela mesma letra minúscula na linha, comparando as biomassas fresca e seca das espécies de fisális dentro de cada substrato, e maiúscula na coluna comparando os substratos e biomassas fresca e seca para cada espécie de fisális, não diferem entre si pelo teste de Skott-Knott, a 5\% de probabilidade de erro.

A disponibilidade de matéria orgânica e nutrientes do substrato à planta é considerada essencial ao seu desenvolvimento, por estar ligada à formação de esqueletos carbônicos, o que garante o acúmulo de biomassa vegetal (OLIVEIRA \& HERNANDEZ 2008).

Os resultados encontrados no presente trabalho são satisfatórios, porém, estudos futuros e mais abrangentes devem ser realizados a respeito de técnicas de cultivo e biologia das espécies do gênero Physalis, a fim de tornar a cultura no Brasil economicamente e racionalmente viável.

\section{CONCLUSÃO}

Ambiente protegido e telado propicia melhor germinação de sementes de Physalis ixocarpa e Physalis minima. 
Crescimento inicial de mudas de $P$. ixocarpa deve ser produzido em ambiente protegido, utilizando como mistura de substrato, Húmus Fértil ${ }^{\circledR}+$ vermiculita expandida de textura média ou Latossolo + vermiculita.

Crescimento inicial de mudas de $P$. minima deve ser produzido em mistura de Húmus Fértil ${ }^{\circledR}+$ vermiculita, sob ambiente protegido ou telado.

\section{REFERÊNCIAS}

ANDRIOLO JL. 2011. Fisiologia das culturas protegidas. 1.ed. Santa Maria: Editora UFSM. 142p.

BRAUN H et al. 2010. Produção de mudas de tomateiro por estaquia: efeito do substrato e comprimento de estacas. Idesia 28: 9-15.

CARVALHO NM \& NAKAGAWA J. 2000. Sementes: ciência, tecnologia e produção. 4.ed. Jaboticabal: FUNEP. 588p. CAVIGLIONE JH et al. 2000. Cartas climáticas do Paraná. Edição 2000, versão 1.0. Londrina: IAPAR. CD ROM.

FACHINELLO JC et al. 2005. Propagação de Plantas Frutíferas. Brasília: Embrapa. 221p.

FERNANDES $C$ et al. 2006. Desempenho de substratos no cultivo do tomateiro do grupo cereja. Horticultura Brasileira 24: $42-46$.

FERRAZ MV et al. 2005. Caracterização física e química de alguns substratos comerciais. Acta Scientiarum. Agronomy 27: 209-214.

FERREIRA DF. 2011. Sisvar: a computer statistical analysis system. Ciência e Agrotecnologia. 35: 1039-1042.

GENDREAU E \& CORBINEAU F. 2009. Physiological aspects of seed dormancy in woody ornamental plants. Propagation of Ornamental Plants 9: 151-158.

HARTMANN HT et al. 2011. Plant propagation: principles and practices. 8.ed. New York: Englewood Clipps. 915p.

KRATZ D et al. 2013. Propriedades físicas e químicas de substratos renováveis. Revista Árvore 37: 1103-1113.

LIMA CSM et al. 2010. Sistemas de tutoramento e épocas de transplante de physalis. Ciência Rural 40: 2472-2479.

LIMA JD et al. 2008. Efeitos da luminosidade no crescimento de mudas de Caesalpinia ferrea Mart. ex Tul. (Leguminosae, Caesalpinoideae). Acta Amazonica 38: 5-10.

LIMA JUNIOR EC et al. 2005. Trocas gasosas, características das folhas e crescimento de plantas jovens de Cupania vernalis Camb. submetidas a diferentes níveis de sombreamento. Ciência Rural 35: 1092-1097.

MAGUIRE JD. 1962. Speed of germination: aid in selection and evaluation for seedling emergence and vigor. Crop Science 2: 176-177.

MARCOS FILHO J. 2005. Fisiologia de sementes de plantas cultivadas. Piracicaba: FEALQ. 495p.

MEIRA MR et al. 2012. Crescimento, produção de fitomassa e teor de óleo essencial de melissa (Melissa officinalis L.) sob diferentes níveis de sombreamento. Revista Brasileira de Plantas Medicinais 14: 352-357.

MUNIZ J et al. 2014. General aspects of physalis cultivation. Ciência Rural 44: 964-970.

OLIVEIRA AB et al. 2009. Emergência de plântulas de Copernicia hospita Martius em função do tamanho da semente, do substrato e ambiente. Revista Brasileira de Sementes 31: 281-287.

OLIVEIRA AB \& HERNANDEZ FFF. 2008. Absorção de nutrientes em mudas de berinjelas cultivadas em substratos alternativos. Revista Ciência Agronômica 39: 583-589.

PELIZZA TR et al. 2013. Produção de mudas de meloeiro amarelo, sob cultivo protegido, em diferentes substratos. Revista Ceres 60: 257-261.

PIVA AL et al. 2013. Emergence and initial development of Cape gooseberry (Physalis peruviana) seedlings with different substrate composition. African Journal of Agricultural Research 8: 6579-6584.

REIS JMR et al. 2010. Estratificação, ambientes e giberelina na antecipação da enxertia do pessegueiro 'Okinawa'. Bioscience Journal 26: 591-601.

RODRIGUES E et al. 2009. Minerals and essential fatty acids of the exotic fruit Physalis peruviana L. Ciência e Tecnologia de Alimentos 29: 642-645.

RUFATO L et al. 2008. Aspectos técnicos da cultura da Physalis. 1.ed. Lages: CAV/UDESC; Pelotas: UFPel. 100p.

SBRUSSI CAG et al. 2014. Maturation stages of fruit development and physiological seed quality in Physalis peruviana. Revista Ciência Agronômica 45: 543-549.

SILVA DF et al. 2013. Conservação pós-colheita de fisális e desempenho produtivo em condições edafoclimáticas de Minas Gerais. Revista Ceres 60: 826-832.

SILVA DF et al. 2016. The production of Physalis spp. seedlings grown under different-colored shade nets. Acta Scientiarum. Agronomy 38: 257-263.

SILVA DF et al. 2017. Viabilidade polínica e quantificação de grãos de pólen em espécies de fisális. Revista Ciência Agronômica 48: 365-373.

SOUZA GS et al. 2014. Crescimento vegetativo e produção de óleo essencial de plantas de alecrim cultivadas sob telas coloridas. Bioscience Journal 30: 232-239.

VIDA JB et al. 2004. Manejo de doenças de plantas em cultivo protegido. Fitopatologia brasileira 29: 355-372. 